\title{
Una experiencia de coordinación dirigida a trabajar las competencias necesarias para superar con éxito el Trabajo de Fin de Grado en Derecho
}

\section{Ixusko Ordeñana Gezuraga}

Profesor Derecho Procesal Universidad del País Vasco, ixusko.ordenana@ehu.eus

\begin{abstract}
An experience of teaching innovation that 16 professors of Law Faculty of the University of the Basque Country are implementing is taken to IV National Congress on Educational Innovation and Network Teaching. Concerned about troubles that students perceive to overcome their Final Degree Project, which is linked to the basic competences of the jurist, a coordinated action has been proposed to work on them, inspired by active and autonomous learning. The origins and basis of this experience are explained.
\end{abstract}

\section{Keywords:}

teaching innovation project, Final Degree Project, competences, active and autonomous learning

\section{Resumen}

Se presenta al IV Congreso Nacional de Innovación Educativa y Docencia en Red la experiencia de innovación docente que están implementando 16 profesores y profesoras de la Facultad de Derecho de la Universidad del País Vasco. Preocupados por los problemas que advierten en el estudiantado para superar el Trabajo Fin de Grado, que se vincula con las competencias básicas del jurista, se han planteado una acción coordinada para trabajarlas, bebiendo, al efecto, del aprendizaje activo y autónomo. Se explican los orígenes y fundamento del mismo.

\section{Palabras clave:}

Proyecto de innovación docente, Trabajo Fin de Grado, competencias, aprendizaje activo y autónomo 


\section{Introducción}

Más de un lustro después del pleno desarrollo del Grado en Derecho, en el marco del Espacio Europeo de Educación Superior, en la Facultad de Derecho de la Universidad del País Vasco-Euskal Herriko Unibertsitatea ${ }^{1}$, el profesorado hemos tenido ocasión de constatar las ventajas y debilidades del nuevo Plan de estudios. En nuestro empeño de implementar con éxito nuevas formas y métodos de enseñanza-aprendizaje, adecuados a las exigencias actuales, en el contexto del aprendizaje cooperativo y dinámico ${ }^{2}, 16$ profesores de la Facultad, encargados de todas las materias que integran el plan de estudios, hemos puesto en marcha un proyecto de innovación educativa centrado en, lo que a nuestro juicio, es una debilidad de nuestro Grado, en general, y del método de enseñanza-aprendizaje que estamos implementando, en particular: el estudiantado llega al último curso del Grado sin dominar las competencias necesarias para realizar y defender el Trabajo Fin de Grado ${ }^{3}$. Queremos aprovechar el IV Congreso Nacional de Innovación Educativa y Docencia en $\operatorname{Red}^{4}$ para relatar nuestro proyecto y experiencia, desde la premisa de que, seguramente, nuestro "problema" y las posibles soluciones que planteamos para el mismo, se repiten en otros Grados y otras Facultades. Al mismo tiempo, nos mueve el interés de socializar nuestro proyecto de intervención en el aula, conscientes de que la opinión que sobre el mismo nos pueden aportar otros docentes pueden ayudar a enriquecerlo y optimizar su resultado.

Una última nota introductoria: el proyecto lleva a penas un curso en marcha, por lo que vamos a compartir su planteamiento y lo implementado hasta la fecha, sin perjuicio de que nos comprometemos a acudir al IN-RED 2019 para relatar su culminación.

\section{Objetivos}

Ante el "problema" planteado -que nosotros y nosotras siempre hemos leido como reto-, el grupo de profesores mentado, integrado por personas con más y menos experiencia en innovación, bajo la coordinación del profesor Ordeñana, nos hemos unido en el proyecto que les vamos a relatar para aunar esfuerzos con los siguientes objetivos principales:

1. Constatar objetivamente la cuestión, ahondando en sus causas.

2. Diseñar un programa o plan de actuación que abarque todas las asignaturas del Grado en Derecho, basado en el aprendizaje activo y autónomo.

3. Implementar el plan de actuación o intervención en el aula, dirigido a obtener las competencias necesarias para superar con éxito el TFG.

\footnotetext{
${ }^{1}$ En lo sucesivo, UPV-EHU.

2 En el marco del modelo pedagógico instaurado por la UPV/EHU, "Irakaskuntza Kooperatiboa eta Dinamikoa” (IKD) (en castellano, "Enseñanza Cooperativa y Dinámica”)

${ }^{3}$ En lo sucesivo, TFG.

${ }^{4}$ En lo sucesivo, IN-RED 2018
}

(cc) EY-Nc-No 2018, Universitat Politècnica de València

Congreso IN-RED (2018) 
4. Que nuestro alumnado no conciba el TFG como algo ajeno al resto de materias que integran el Grado en Derecho. Lejos de ello, aspiramos a que lo sienta no sólo como parte, sino como culmen del proceso de enseñanza-aprendizaje vinculado al Grado en Derecho, tarea que le permite "demostrar haber adquirido los contenidos formativos, capacidades, competencias y habilidades para los que le capacita la correspondiente titulación” (art. 1.3. Reglamento para la elaboración y defensa de los TFG de la Facultad de Derecho de la UPV/EHU) ${ }^{5}$. Por ello, pretendemos que, lejos de verlo como obstáculo (último) para obtener la Graduación, lo perciban como ejercicio de afianzamiento del conocimiento y competencias adquiridas.

5. Objetivamente, nos daríamos por satisfechos si la mayoría de nuestro alumnado (un 70\%) defendiera el TFG en la primera convocatoria del año de matriculación y obtuviera como media un 7,5. Ambos indicadores de aprendizaje reflejarían una mejora considerable de la realidad actual.

Todo ello guiado con la noble intención de mejorar el aprendizaje del estudiantado de Derecho en la adquisición de competencias básicas de la titulación. Efectivamente, el TFG, integrado en el cuarto módulo del Grado en Derecho de la Facultad de Derecho de la UPV/EHU -Especialización, Investigación y Práctica del Derecho-, persigue asentar competencias genéricas y transversales de la titulación ${ }^{6}$, entre las que cabe destacar: 1) la capacidad de análisis crítico, interrelación e integración de conocimientos jurídicos; 2) la comunicación oral y escrita; 3) la capacidad de síntesis y exposición, con utilización de técnicas de argumentación y razonamiento crítico y 4) la gestión del propio proceso de aprendizaje hacia una formación integral y respetuosa con los valores democráticos.

Las primeras tres competencias apuntadas se trabajan, igualmente, en distintas asignaturas de los tres módulos restantes del Grado. La última, por su parte, casa a la perfección con la metodología de enseñanza-aprendizaje que quiere impulsar y emplear el proyecto educativo que se propone: el aprendizaje activo y autónomo del alumnado.

\section{Desarrollo de la innovación}

\subsection{Sobre su origen}

El germen primario del proyecto de innovación educativa que estamos describiendo son muchas conversaciones -formales e informales- entre los profesores y profesoras integrantes del grupo los últimos años y las consiguientes reflexiones individuales y grupales, previas y posteriores. Todos los miembros del equipo hemos realizado labores de dirección y evaluación de TFGs. Nuestra experiencia de más de 5 años en la materia, nos lleva a entender que "la memoria, estudio, informe o dictamen original vinculado a alguna/s de las materias desarrolladas en el Grado" (art. 1 REDTFG) que debe realizar el estudiante para culminar el Grado en Derecho se ha convertido para muchos estudiantes

\footnotetext{
${ }^{5}$ En lo sucesivo, REDTFG

${ }^{6}$ En estos conceptos ahondaremos en breve.
} 
en un trámite muy duro de superar. A pesar de que los 16 docentes integrantes del equipo que propone el proyecto mantenemos que en la enseñanza-aprendizaje de las asignaturas que impartimos trabajamos más o menos directamente las competencias que se vinculan al TFG ((1)capacidad de análisis crítico, interrelación e integración de conocimientos jurídicos; (2) comunicación oral y escrita; (3) capacidad de síntesis y exposición, con utilización de técnicas de argumentación y razonamiento crítico y (4) gestión del propio proceso de aprendizaje hacia una formación integral y respetuosa con los valores democráticos), cuando nos encontramos mano a mano con el alumno o alumna al que tenemos que dirigir el TFG, advertimos que tienen muchas y verdaderas dificultades para realizarlo. Son numerosos los problemas que percibimos: les cuesta elegir y determinar el tema concreto del TFG -algo difícil de entender cuando ya han cursado todas las materias de la carrera-; no son, en general, diestros en la búsqueda de información, mostrando verdaderos apuros para articular y manejar la bibliografía (casi su único recurso es google); les cuesta sintetizar y construir un argumento sistemático, coherente y crítico, y presentarlo, tanto por escrito, como oralmente; y en todo este proceso buscan en nosotros y nosotras un padre o madre que apruebe cada una de sus decisiones y opiniones (¿dónde está la gestión del propio proceso de aprendizaje que se les supone para estas alturas del Grado?). Sin duda alguna, todas ellas son competencias que, al final del TFG, deben obtener pero que parece que previamente no han adquirido.

\subsection{Constatación del "problema”, mejor llamado "reto"}

Explicado el origen de nuestro proyecto, la primera actuación desarrollada en su implementación ha sido la constatación del "problema". Al efecto, hemos utilizado dos elementos: la estadística y la consulta directa al estudiantado. La primera viene a avalar la preocupación del equipo docente involucrado en el proyecto. Los datos oficiales obtenidos muestran que la mayoría del estudiantado del cuarto curso del Grado en Derecho presenta el TFG en la segunda convocatoria de su año de matriculación. Además, la nota media del ejercicio los dos últimos cursos es un 6,5. Ambos elementos requieren un juicio subjetivo: se nos antoja difícil de entender que la mayoría del alumnado presente su TFG en segunda convocatoria, cuando tienen un cuatrimestre completo vacío, sin asignaturas ${ }^{7}$, para confeccionarlo. No consideramos que sea un problema tanto de tiempo, como de inseguridad. Por otra parte, la nota obtenida en el ejercicio no nos parece excesivametne buena, sino más bien escasa, si advertimos especialmente que las competencias vinculadas con el TFG son esenciales del Grado en Derecho.

Cuestionado el estudiantado, informalmente en el transcurso de los cursos, y más formalmente, con ocasión de la preparación del proyecto de innovación educativa que estamos describiendo -preguntamos a más de 40 estudiantes que el curso pasado (2016/2017) se matricularon para hacer el TFG con nosotros y nosotras- es tajante: no se sienten preparados para afrontar el TFG. No asocian tareas y actividades realizadas

${ }^{7}$ El segundo del cuarto curso.

(c) EY-Nc-ND 2018, Universitat Politècnica de València 
previamente en otras asignaturas con el último ejercicio o requisito para obtener la Graduación. Se sienten inexpertos y muy perdidos.

Por tanto, y resumiendo, entendemos que el proyecto de innovación docente que estamos implementando viene a responder a una necesidad práctica: la superación de los obstáculos que encuentra el alumnado en la realización y superación del TFG, en cuanto forma de garantizar que adquiere sobradamente las competencias básicas que se requieren al jurista. Entendemos, al respecto, el TFG como elemento excelente para ahondar en "la gestión personal del propio aprendizaje” que se le vincula como competencia básica. Para ello, es necesario previamente trabajar consciente y sistemáticamente el aprendizaje activo y autónomo del alumnado.

\subsection{Un plan para fomentar el aprendizaje activo y autónomo del alumnado dirigido a adquirir las competencias necesarias para superar con éxito el TFG.}

\subsubsection{Elementos esenciales del plan.}

Luego, al hilo de lo descrito, el eje pedagógico de la intervención que les estamos relatando es el aprendizaje activo y autónomo del alumnado. Se trata, concretamente, de fomentarlo por los docentes que integran el proyecto, en los cursos previos del Grado de Derecho y antes de la realización del TFG, para que puedan demostrarlo en la confección de éste, salvándolo con éxito. Concebimos la realización y superación del TFG como la mejor evidencia de que el estudiantado ha adquirido las competencias correspondientes mediante el aprendizaje activo y autónomo.

En las próximas líneas vamos a ahondar en el marco teórico y práctico de los elementos esenciales de nuestro plan de actuación: las competencias a adquirir y el aprendizaje activo y autónomo como instrumento, al efecto. Relataremos, por último, la serie de actividades previstas en el marco de nuestro plan de actuación.

\subsubsection{Sobre las competencias a adquirir.}

Alejándonos de discusiones vanas ${ }^{8}$, sin negar su origen del mundo laboral ${ }^{9}$ y que se han convertido en la piedra angular para la confección de los planes de estudios ${ }^{10}$, entendemos por competencia "la capacidad de hacer frente con garantías de éxito a una situación problemática en un determinado contexto"11, integrándose por una operación (acción

\footnotetext{
8 Da fé de ello, GUZMÁN MARÍN, F., “El concepto de competencias”, Revista Iberoamericana de Educación (2012), núm. 4. Iberoamérica: Organización de Estados Iberoamericanos para la Educación, la Ciencia y la Cultura.

9 Incide en ello, LUIS MANRÍQUEZ P. “¿Evaluación en competencias?”, Estudios pedagógicos (2012), núm. 1, Chile: Facultad de Filosofía y Humanidades Universidad Austral de Chile, argumentando que es una característica de la época en la que nos toca vivir. En el mismo sentido, ESTEBAN ALBERT, M., SÁEZ CARRERAS, J., "Las profesiones, las competencias y el mercado”, REDU: Revista de Docencia Universitaria (2008), núm. 2, Valencia: Universidad Politécnica.

${ }^{10}$ Así la califican, con total acierto, DEL CARMEN LEVÍ ORTA, G., RAMOS MÉNDEZ, E., “Componentes de las competencias en los nuevos grados de algunas universidades españolas”, Revista de educación (2013), núm. 362. Madrid: Ministerio de Educacion, Cultura y Deporte.

${ }^{11}$ Definición otorgada por GOÑI ZABALA, J.M. (2005), El Espacio Europeo de Educación Superior, un reto para la Universidad. Barcelona: Octaedro, p. 105 y ss. En sentido similar, ZABALZA, M.A. (2009), Competencias docentes del profesorado universitario. Calidad y desarrollo profesional, 4. ed. Madrid: Narcea.
}

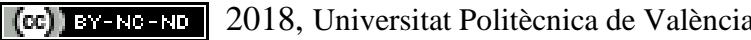


mental) sobre un objeto (que es lo que habitualmente llamamos conocimiento) para el logro de un fin determinado (contexto de aplicación).

Las competencias, incidiendo en su tipología, pueden ser generales o de titulación y específicas.

Denominamos competencia general o de titulación a aquélla que debe adquirir el estudiante al finalizar el Grado. Estas competencias se escalonan por cursos o módulos, integrando su conjunto las características descritas en el perfil del graduado. En el marco de las competencias generales debemos destacar una categoría especial: las competencias transversales. Llamamos así a aquéllas que deben ser trabajadas en todos los niveles inferiores a aquél en que está ubicada ${ }^{12}$.

Por su parte, denominamos competencia específica a aquélla correspondiente a cada módulo y/o asignatura.

Pasando del plano meramente teórico al práctico, ¿Cuáles son las competencias generales a adquirir por los alumnos en el Grado en Derecho de la UPV-EHU? Son 5:

1) Poseer y comprender las bases terminológicas y de conocimiento fundamental del Derecho Público y del Privado con plena capacidad de sistematización, análisis crítico, interrelación, integración y síntesis, con espíritu de liderazgo y emprendizaje.

2) Aplicar adecuadamente los conocimientos y técnicas adquiridos a lo largo de sus estudios de grado en orden a la correcta resolución de los problemas jurídicos, adoptando decisiones de manera creativa y afrontando situaciones diversas, con respeto a la diversidad y la tolerancia, apoyado en una suficiente capacidad de organización, trabajo en pequeños grupos y planificación.

3) Adaptarse permanentemente a los cambios normativos y a las situaciones novedosas con el imprescindible compromiso ético y la sensibilidad social, económica y medioambiental, en general, primando los aspectos de calidad e innovación, de modo específico, en el ámbito de las TIC.

4) Gestionar satisfactoriamente las relaciones interpersonales y sociales, a partir del oportuno dominio de los procesos de comunicación oral y escrita que faculte al alumnado para transmitir información, tanto a un público especializado, como al no especializado, utilizando técnicas de argumentación y razonamiento crítico.

5) Gestionar el proceso de aprendizaje de modo continuado y autónomo, de modo que se obtenga una formación integral coherente con los derechos fundamentales de igualdad, no discriminación y fomento de la cultura de la paz, y así poder afrontar con éxito estudios posteriores de especialización o reciclaje.

Estas competencias las ha de adquirir el egresado en Derecho poco a poco, escalonadamente, mientras estudia las diferentes asignaturas que integran los cuatro módulos en los que se estructura la carrera ${ }^{13}$. Y, en su contexto, tenemos que entender las competencias específicas -ya expuestas- que se vinculan al TFG. Con todo, entendemos que la obtención por el alumnado de estas competencias exige al profesorado "poner en práctica

\footnotetext{
${ }^{12}$ Muestra otras definiciones GARCÍA MARTÍNEZ, J. (2007), “Las competencias y la nueva organización de la enseñanza”, en AAVV (Coor. LEÓN BENÍTEZ, M.R.), La Licenciatura de Derecho en el contexto de la convergencia europea. Valencia: Tirant lo Blanch, pp. 168-173.

${ }^{13}$ Los cuatro módulos son: (1) las bases del derecho, (2) el derecho público general y especial, (3) el derecho privado, y (4) especialización, investigación y práctica del derecho
}

(cc) EY-NC-ND 2018, Universitat Politècnica de València 
nuevas pedagogías coherentes con el sentido y significado de dichas competencias” ${ }^{14}$. De ahí el proyecto que les relatamos.

\subsubsection{La forma de adquirir las competencias vinculadas con el TFG: el aprendizaje activo} y autónomo.

Entendemos que la forma o recurso pedagógico idóneo para que el estudiantado adquiera las competencias apuntadas es el aprendizaje activo y autónomo ${ }^{15}$. Planteamos beber de esta fuente conscientemente para mejorar el proceso enseñanza-aprendizaje.

En el marco del aprendizaje activo ${ }^{16}$, entendemos imprescindible utilizar estrategias en nuestra docencia que involucren dinamicamente al estudiantado, para que obtengan una comprensión más profunda de los contenidos, que les permita desarrollar un pensamiento crítico y habilidades para solventar problemas (análisis, síntesis, argumentación y defensa), al tiempo que les motivemos, y animemos a la interacción y trabajo con sus semejantes ${ }^{17}$. Entendemos necesario, al efecto, resignificar los roles de los propios estudiantes y el nuestro, otorgando al alumando el protagonismo máximo que se merece en el proceso ${ }^{18}$. Tenemos que configurar, asimismo, un nuevo concepto del lugar de trabajo: aula o espacio ajeno a ella ${ }^{19}$.

En el proceso descrito, totalmente vinculado, deviene igual de importante lo que se viene llamando aprendizaje autónomo, autoaprendizaje o estudio autodirigido. Es necesario trabajar con los y las estudiantes la capacidad de detectar déficits en el propio conocimiento y superarlas mediante la reflexión crítica y la elección de la mejor actuación al respecto. Y es que el aprendizaje autónomo "implica por parte del que aprende asumir la responsabilidad y el control interno del proceso personal de aprendizaje” ${ }^{20}$.

\footnotetext{
${ }^{14}$ Literal, GOYES MORENO, I. (2013)“Competencias pedagógicas para la formación jurídica por competencias”, Academia: revista sobre enseñanza del derecho de Buenos Aires, núm. 21. Buenos Aires: Facultad de Derecho de la Universidad de Buenos Aires.

${ }^{15}$ Es más, justifica que esta forma de aprendizaje se corresponde con la dimensión actitudinal de las competencias, RIBAS FERRER, V. (2011) “El aprendizaje por competencias y su coordinación en los estudios de Derecho”, Anuario de la Facultad de Derecho (Universidad de Alcalá), núm. 4. Madrid: Universidad de Alcalá.

${ }^{16}$ Exponen sus fundamentos en aplicación a una experiencia real, VIDAL SALAZAR, M.D., FERRÓN VÍLCHEZ, V., LEYVA DE LA HIZ, D.I. (2014), “Promoviendo el aprendizaje activo del alumnado universitario mediante el trabajo autónomo”, AAVV, Prácticas de innovación docente en ciencias sociales y jurídicas. Granada: Comares, pp. 41-47.

${ }^{17}$ Remarca la importancia de que el alumno vaya tomando decisiones, PÉREZ DE ALBÉNIZ ITURRIAGA, A. (2015), et al, “Metacognición en un proceso de aprendizaje autónomo y cooperativo en el aula universitaria”, Contextos educativos: Revista de educación, núm. 18. La Rioja: Universidad de La Rioja. Nos movemos, por otra parte, en los postulados de lo que los norteamericanos denominan cooperative learning (aprendizaje cooperativo). Sobre sus características, ORDEÑANA GEZURAGA, I. (2010), "Mediation: an adequate tool to solve consumer dispute", AAVV (Ed. IATED), EDULEARN 2010 Proceedings. Barcelona: IATED.

${ }^{18}$ En este sentido, TEJADA FERNÁNDEZ, J. (2002), "El docente universitario ante los nuevos escenarios: implicaciones para la innovación docente”, Acción Pedagógica, núm. 2. Venezuela: Universidad de los Andes.

${ }^{19}$ No en vano, con la configuración actual y vigente, cada crédito de las asignaturas supone 10 horas de docencia en el aula y 15 de trabajo externo.

${ }^{20}$ Literal, con mucho soporte bibliográfico, ESCRIBANO GONZÁLEZ, A. (1995), “Aprendizaje cooperativo y autónomo en la enseñanza universitaria”, Enseñanza \& Teaching: Revista interuniversitaria de didáctica, núm. 13. Salamanca: Universidad de Salamanca.
}

(c)) EY-NC-ND 2018, Universitat Politècnica de València 


\subsubsection{Líneas básicas del plan.}

Con las premisas expuestas, los 16 miembros del equipo del proyecto de innovación docente hemos planificado una serie de actividades divididas por cursos y asignaturas, basadas en la metodología del aprendizaje activo y autónomo para trabajar (y adquirir, obviamente) las competencias vinculadas al TFG, que -repetimos- son, al tiempo, esenciales en el Grado en Derecho.

Estamos ante un plan o sistema de actuación que abarca todos los módulos, cursos y materias del Grado en Derecho; así tiene que ser pues pretendemos entrenar conscientemente a nuestros alumnos y alumnas para realizar y superar sin problema alguno el TFG, por ser ello la mejor evidencia de que, además de gestionar su propio aprendizaje, detentan las competencias más importantes del jurista.

En este marco el equipo docente vamos a convertirnos en meros facilitadores o instructores, ayudando a los y las estudiantes a buscar los instrumentos necesarios para superar las dificultades que se les planteen. Deben ser ellos los protagonistas de su aprendizaje, adquiriendo un rol o intervención primordial en el establecimiento de los objetivos, recursos, procedimientos, y evaluación del mismo. Deben partir de sus conocimientos actuales, para con nuestra ayuda, construir los futuros. Sólo así estarán preparados para ser los verdaderos protagonistas de su TFG.

Esta planificación se ha realizado teniendo en cuenta los siguientes criterios:

a)Realidades de las asignaturas concretas (curso, metodología, temario, nivel de dificultad,...)

b)Necesidades y capacidades del estudiantado.

c)Características de los grupos de alumnado.

d)Tiempo dedicable al proyecto de innovación educativa.

En aplicación de los mismos, se ha programado una actividad por cada asignatura de los tres módulos precedentes dirigida directamente a preparar la confección del TFG, trabajando conscientemente las competencias que requiere éste. Todas las actividades están coordinadas y directamente relacionadas. Cabe apuntar, además, que conforme a nuestra planificación todo el alumnado del Grado en Derecho -sin perjuicio del curso en el que se encuentre, y por tanto, más o menos cerca del TFG- va a estar trabajando consciente y coordinadamente las competencias básicas del Grado, que coinciden básicamente con las vinculadas al TFG.

En el primer curso, en las tres asignaturas involucradas en el proyecto (Historia del Derecho, Derecho Romano y Teoría del Derecho), se van realizar tareas de síntesis y esquematización de sentencias y textos jurídicos básicos, presentes y pasados, en grupo e individualmente. Así mientras en Historia del Derecho se les va a pedir que hagan un resumen del contenido del Fuero Viejo de Vizcaya, en Derecho Romano van a esquematizar las Leyes Licinias.Por su parte, en Teoría del Derecho van a leer y resumir una sentencia del Tribunal Constitucional. En las tres asignaturas se va a hacer especial hincapié en que la capacidad de comprensión y síntensis es esencial en el Grado en Derecho. Únicamente la actividad de Teoría del Derecho será individual; las otras dos serán grupales.

Con mayor exigencia y dedicación, el segundo curso, en Introducción al Derecho Procesal, Derecho Administrativo, Derecho Internacional Público y Derecho Constitucional, en el marco del proyecto de innovación docente que estamos describiendo, 
el alumnado va a redactar, en grupo, textos jurídicos relativos a cuestiones controvertidas actuales (independencia judicial, déficit y servicios públicos, guerras olvidadas, modificación constitucional...) para, posteriormente, defenderlos en un debate público. Concretamente, en Introducción al Derecho Procesal se va a trabajar sobre una noticia periodística en relación a la objetividad judicial; en Derecho Administrativo un bando municipal se va a convertir en la excusa para que el estudiantado presente alegaciones en contra del mismo; en Derecho Internacional Público van analizar la situación del Yemen, para remitir a la ONU misivas solicitando que se ponga atención a la guerra olvidada que vive este país. Por último, en Derecho Constitucional, se va a proponer, por escrito, una reforma constitucional, dividiendo, al efecto, al alumnado en grupos correspondientes al arco de partidos políticos existentes en España. En todas las actividades, además de articular un texto grupal con un índice, cuerpo central y conclusiones, el estudiantado tendrá que defender su contenido en un debate público colectivo, actuando como moderador/a el docente correspondiente.

Superado el ecuador del Grado en Derecho, el tercer curso, al tiempo que los estudiantes trabajan las asignaturas más jurídicas (Derecho Procesal Civil, Derecho Procesal Penal, Derecho Penal, Derecho Laboral y Derecho Internacional Privado) aprovecharemos las clases prácticas de las mismas para que configuren escritos de posiciones diversas y textos judiciales, en grupo e individualmente. A todos ellos, les seguirán defensas públicas, individuales y grupales, y los consiguientes debates. Concretamente, en Derecho Procesal Civil, redactarán grupalmente un escrito de demanda y en Derecho Procesal Penal, individualmente, un escrito de acusación. En Derecho Penal realizarán escritos de calificación individualmente y en Derecho Laboral redactarán un convenio colectivo en grupo. Por último, en Derecho Internacional Privado, individualmente, cada alumno se pondrá en el lugar de un letrado/a que solicita alimentos en el supuesto de un divorcio internacional. Todos los profesores y profesoras implicados en este proyecto de innovación educativa encargados de estas asignaturas harán expresamente hincapié al alumando en la necesidad de trabajar la capacidad de análisis crítico, interrelación e integración de conocimientos jurídicos; la comunicación oral y escrita; y la capacidad de síntesis y exposición, con utilización de técnicas de argumentación y razonamiento crítico. Se les exigirá, al tiempo ,que sean capaces de gestionar el propio proceso de aprendizaje, tomando las decisiones pertinentes de forma madura, coherente y crítica.

Ya, el último curso, el primer cuatrimestre, y antes de realizar el TFG, en el segundo, en el transcurso de las últimas asignaturas del Plan de estudios (Derecho Financiero, Derecho Civil IV y Derecho Mercantil) se realizarán breves trabajos de investigación y defensa pública sobre cuestiones no estudiadas en el aula, pero directamente relacionadas con ellas, sobre temas planteados por el propio alumnado. Se trata de emular la actividad que va a exigir el TFG a continuación, sirviendo de entrenamiento -mejor calentamiento- previo. En los tres casos se va a seguir la misma dinámica exigida para los TFGs, si bien adaptada a su condición de ejercicio preparatorio (la extensión va a ser dos tercios menos que la exigida al TFG; las tutorías de preparación van a ser colectivas y las defensas en el aula y con posibilidad de intervención del resto de alumnado).

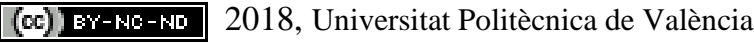




\section{Resultados}

Con el proyecto de intervención en el aula que les estamos describiendo vivo y sin culminar, con las actividades desarrolladas hasta el momento, podemos destacar los siguientes resultados:

1. Constatación clara del problema, que hemos convertido en reto: la preocupación y sospecha inicial del equipo docente involucrado (las taras del alumnado para encarar el TFG) ha venido avalada por las primeras fases del proyecto, en general, y en especial, por la constatación, de manera objetiva, de las debilidades que muestra el estudiantado cuando se enfrenta al TFG.

2. Pleno acierto en la puesta en marcha del proyecto de innovación docente: el reto que nos planteamos exige una actividad conjunta y coordinada del claustro del Grado en Derecho de la Facultad de Derecho de la UPV/EHU. Se han dado los primeros pasos, al respecto, y en lo consiguiente, se realizarán los posteriores encaminados a obtener los resultados de aprendizaje mentados.

3. Planificación completa, sistemática y coordinada de una serie de actividades a desarrollar en el marco de todos los módulos que integran el Grado en Derecho, a lo largo de sus cuatro cursos: el proyecto que les hemos descrito tiene el valor fundamental de suponer la primera ocasión en que todas las asignaturas que integran el plan de estudios del Grado en Derecho se coordinan dirigidas a obtener un resultado común. Ha sido esencial, en este sentido, la labor -y la paciencia- de los 16 profesores y profesoras involucrados en el proyecto. Tras muchas discusiones y debates, hemos conseguido configurar un proyecto común que comparte objetivos (adquirir las competencias mentadas) y metodología (aprendizaje activo y autónomo)

\section{Conclusiones}

En el momento en el que nos encontramos en la implementación del proyecto de innovación docente que les hemos descrito, en este prestigioso foro del IN-RED 2018, y desde la perspectiva de la coordinación del mismo, queremos presentar las siguientes conclusiones:

1. El equipo docente involucrado en el proyecto entiende esenciales para el jurista las competencias que se vinculan al TFG: 1)capacidad de análisis crítico, interrelación e integración de conocimientos jurídicos; 2)capacidad de comunicación oral y escrita; 3) capacidad de síntesis y exposición, con utilización de técnicas de argumentación y razonamiento crítico y 4) gestión del propio proceso de aprendizaje hacia una formación integral y respetuosa con los valores democráticos. Son las competencias aceptadas por el ANECA al verificar el plan de estudios del Grado en Derecho de la UPV/EHU y que, al tiempo, entendemos imprescindibles en la formación del futuro jurista.

2. El equipo docente involucrado y el alumnado consultado avienen que, en la actualidad, en los años que lleva en vigor el nuevo plan de estudios del Grado en Derecho, de la mano del Espacio Educativo Europeo Superior, no se está garantizando la adquisición plena, por parte del estudiantado, de las competencias mentadas.

3. Por ende, resulta esencial la intervención en el aula, al efecto. El proyecto de innovación educativa que se ha descrito está llamado a garantizar que el futuro jurista adquiere competencias que le son esenciales y que se vinculan especialmente con el TFG.

(cc) EY-NC-ND 2018, Universitat Politècnica de València

Congreso IN-RED (2018) 
4. La intervención mentada no puede ser aislado o individual, más debe ser colectiva, sistemática y coordinada.

5. El aprendizaje activo y autónomo consciente y planificado puede ser la metodología adecuada para que el estudiantado adquiera las competencias mentadas.

\section{Referencias}

DEL CARMEN LEVí ORTA, G., RAMOS MÉNDEZ, E., "Componentes de las competencias en los nuevos grados de algunas universidades españolas", Revista de educación (2013), núm. 362. Madrid: Ministerio de Educacion, Cultura y Deporte.

ESCRIBANO GONZÁLEZ, A. (1995), “Aprendizaje cooperativo y autónomo en la enseñanza universitaria”, Enseñanza \& Teaching: Revista interuniversitaria de didáctica, núm. 13. Salamanca: Universidad de Salamanca.

ESTEBAN ALBERT, M., SÁEZ CARRERAS, J., "Las profesiones, las competencias y el mercado", REDU: Revista de Docencia Universitaria (2008), núm. 2. Valencia: Universidad Politécnica.

GARCÍA MARTÍNEZ, J. (2007), "Las competencias y la nueva organización de la enseñanza”, en AAVV (Coor. LEÓN BENÍTEZ, M.R.), La Licenciatura de Derecho en el contexto de la convergencia europea. Valencia: Tirant lo Blanch, pp. 168-173.

GOÑI ZABALA, J.M. (2005), El Espacio Europeo de Educación Superior, un reto para la Universidad. Barcelona: Octaedro, p. 105 y ss.

GOYES MORENO, I. (2013), “Competencias pedagógicas para la formación jurídica por competencias”, Academia: revista sobre enseñanza del derecho de Buenos Aires, núm. 21. Buenos Aires: Facultad de Derecho de la Universidad de Buenos Aires.

GUZMÁN MARÍN, F., "El concepto de competencias", Revista Iberoamericana de Educación (2012), núm. 4. Iberoamérica: Organización de Estados Iberoamericanos para la Educación, la Ciencia y la Cultura.

LUIS MANRÍQUEZ P. “¿Evaluación en competencias?”, Estudios pedagógicos (2012), núm. 1, Chile: Facultad de Filosofía y Humanidades Universidad Austral de Chile.

ORDEÑANA GEZURAGA, I. (2010), "Mediation: an adequate tool to solve consumer dispute”, AAVV (Ed. IATED), EDULEARN 2010 Proceedings. Barcelona: IATED

PÉREZ DE ALBÉNIZ ITURRIAGA, A. (2015), et al, “Metacognición en un proceso de aprendizaje autónomo y cooperativo en el aula universitaria", Contextos educativos: Revista de educación, núm. 18. La Rioja: Universidad de La Rioja.

RIBAS FERRER, V. (2011) "El aprendizaje por competencias y su coordinación en los estudios de Derecho”, Anuario de la Facultad de Derecho (Universidad de Alcalá), núm. 4. Madrid: Universidad de Alcalá.

TEJADA FERNÁNDEZ, J. (2002), "El docente universitario ante los nuevos escenarios: implicaciones para la innovación docente”, Acción Pedagógica, núm. 2. Venezuela: Universidad de los Andes. 
Una experiencia de coordinación dirigida a trabajar las competencias necesarias para superar con éxito el Trabajo de Fin de Grado en Derecho

VIDAL SALAZAR, M.D., FERRÓN VÍLCHEZ, V., LEYVA DE LA HIZ, D.I. (2014), "Promoviendo el aprendizaje activo del alumnado universitario mediante el trabajo autónomo”, AAVV, Prácticas de innovación docente en ciencias sociales y jurídicas. Granada: Comares, pp. 41-47.

ZABALZA, M.A. (2009), Competencias docentes del profesorado universitario. Calidad y desarrollo profesional, 4. ed. Madrid: Narcea.

(c) EY-NC-ND 2018, Universitat Politècnica de València 